\title{
Unification on Subvarieties of Pseudocomplemented Distributive Lattices
}

\author{
Leonardo Cabrer
}

\begin{abstract}
In this paper subvarieties of pseudocomplemented distributive lattices are classified by their unification type. We determine the unification type of every particular unification problem in each subvariety of pseudocomplemented distributive lattices.
\end{abstract}

\section{Introduction}

Syntactic unification theory is concerned with the problem of finding a substitution that equalizes a finite set of pairs of terms simultaneously. More precisely, given a set of function symbols $\mathscr{L}$ and a finite set of pairs of $\mathscr{L}$-terms $U=\left\{\left(t_{1}, s_{1}\right), \ldots,\left(t_{m}, s_{m}\right)\right\}$, called a unification problem, a unifier for $U$ is a substitution $\sigma$ defined on the set of variables of the terms in $U$ such that $\sigma\left(t_{i}\right)=\sigma\left(s_{i}\right)$ for each $i \in\{1, \ldots, m\}$. In many applications the operations in $\mathscr{L}$ are assumed to satisfy certain conditions that can be expressed by equations, such as associativity, commutativity, and idempotency. Then syntactic unification evolves into equational unification. Given an equational theory $E$ in the language $\mathscr{L}$, a unifier for $U$ is now asked to send the terms in each pair $\left(t_{i}, s_{i}\right) \in U$ to terms $\sigma\left(t_{i}\right)$ and $\sigma\left(s_{i}\right)$ that are equivalent for $E$ (in symbols, $\sigma\left(t_{i}\right) \approx_{E} \sigma\left(s_{i}\right)$ ).

Once a particular unification problem is known to admit $E$-unifiers, the next task is to find a complete description of its unifiers. For that we first observe that if $\sigma$ is an $E$-unifier for $U$, then $\gamma \circ \sigma$ is also an $E$-unifier for $U$, whenever $\gamma$ is a substitution such that $\gamma \circ \sigma$ is well defined. In this case, we say that $\sigma$ is more general than $\gamma \circ \sigma$. Therefore, a useful way to determine all the unifiers of a particular problem is to calculate a family of unifiers such that any other unifier of the problem is less general

Received June 11, 2012; accepted February 10, 2014

First published online September 12, 2016

2010 Mathematics Subject Classification: Primary 06D15; Secondary 08B30, 06D50, 03G10

Keywords: unification type, equational unification, projective algebra, pseudocomplemented lattice, topological duality

C 2016 by University of Notre Dame $\quad 10.1215 / 00294527-3659307$ 


\title{
Unification on Subvarieties of Pseudocomplemented Distributive Lattices
}

\author{
Leonardo Cabrer
}

\begin{abstract}
In this paper subvarieties of pseudocomplemented distributive lattices are classified by their unification type. We determine the unification type of every particular unification problem in each subvariety of pseudocomplemented distributive lattices.
\end{abstract}

\section{Introduction}

Syntactic unification theory is concerned with the problem of finding a substitution that equalizes a finite set of pairs of terms simultaneously. More precisely, given a set of function symbols $\mathscr{L}$ and a finite set of pairs of $\mathscr{L}$-terms $U=\left\{\left(t_{1}, s_{1}\right), \ldots,\left(t_{m}, s_{m}\right)\right\}$, called a unification problem, a unifier for $U$ is a substitution $\sigma$ defined on the set of variables of the terms in $U$ such that $\sigma\left(t_{i}\right)=\sigma\left(s_{i}\right)$ for each $i \in\{1, \ldots, m\}$. In many applications the operations in $\mathscr{L}$ are assumed to satisfy certain conditions that can be expressed by equations, such as associativity, commutativity, and idempotency. Then syntactic unification evolves into equational unification. Given an equational theory $E$ in the language $\mathscr{L}$, a unifier for $U$ is now asked to send the terms in each pair $\left(t_{i}, s_{i}\right) \in U$ to terms $\sigma\left(t_{i}\right)$ and $\sigma\left(s_{i}\right)$ that are equivalent for $E$ (in symbols, $\sigma\left(t_{i}\right) \approx_{E} \sigma\left(s_{i}\right)$ ).

Once a particular unification problem is known to admit $E$-unifiers, the next task is to find a complete description of its unifiers. For that we first observe that if $\sigma$ is an $E$-unifier for $U$, then $\gamma \circ \sigma$ is also an $E$-unifier for $U$, whenever $\gamma$ is a substitution such that $\gamma \circ \sigma$ is well defined. In this case, we say that $\sigma$ is more general than $\gamma \circ \sigma$. Therefore, a useful way to determine all the unifiers of a particular problem is to calculate a family of unifiers such that any other unifier of the problem is less general

Received June 11, 2012; accepted February 10, 2014

First published online September 12, 2016

2010 Mathematics Subject Classification: Primary 06D15; Secondary 08B30, 06D50, 03G10

Keywords: unification type, equational unification, projective algebra, pseudocomplemented lattice, topological duality

C 2016 by University of Notre Dame $\quad 10.1215 / 00294527-3659307$ 


\title{
Unification on Subvarieties of Pseudocomplemented Distributive Lattices
}

\author{
Leonardo Cabrer
}

\begin{abstract}
In this paper subvarieties of pseudocomplemented distributive lattices are classified by their unification type. We determine the unification type of every particular unification problem in each subvariety of pseudocomplemented distributive lattices.
\end{abstract}

\section{Introduction}

Syntactic unification theory is concerned with the problem of finding a substitution that equalizes a finite set of pairs of terms simultaneously. More precisely, given a set of function symbols $\mathscr{L}$ and a finite set of pairs of $\mathscr{L}$-terms $U=\left\{\left(t_{1}, s_{1}\right), \ldots,\left(t_{m}, s_{m}\right)\right\}$, called a unification problem, a unifier for $U$ is a substitution $\sigma$ defined on the set of variables of the terms in $U$ such that $\sigma\left(t_{i}\right)=\sigma\left(s_{i}\right)$ for each $i \in\{1, \ldots, m\}$. In many applications the operations in $\mathscr{L}$ are assumed to satisfy certain conditions that can be expressed by equations, such as associativity, commutativity, and idempotency. Then syntactic unification evolves into equational unification. Given an equational theory $E$ in the language $\mathscr{L}$, a unifier for $U$ is now asked to send the terms in each pair $\left(t_{i}, s_{i}\right) \in U$ to terms $\sigma\left(t_{i}\right)$ and $\sigma\left(s_{i}\right)$ that are equivalent for $E$ (in symbols, $\sigma\left(t_{i}\right) \approx_{E} \sigma\left(s_{i}\right)$ ).

Once a particular unification problem is known to admit $E$-unifiers, the next task is to find a complete description of its unifiers. For that we first observe that if $\sigma$ is an $E$-unifier for $U$, then $\gamma \circ \sigma$ is also an $E$-unifier for $U$, whenever $\gamma$ is a substitution such that $\gamma \circ \sigma$ is well defined. In this case, we say that $\sigma$ is more general than $\gamma \circ \sigma$. Therefore, a useful way to determine all the unifiers of a particular problem is to calculate a family of unifiers such that any other unifier of the problem is less general

Received June 11, 2012; accepted February 10, 2014

First published online September 12, 2016

2010 Mathematics Subject Classification: Primary 06D15; Secondary 08B30, 06D50, 03G10

Keywords: unification type, equational unification, projective algebra, pseudocomplemented lattice, topological duality

C 2016 by University of Notre Dame $\quad 10.1215 / 00294527-3659307$ 


\title{
Unification on Subvarieties of Pseudocomplemented Distributive Lattices
}

\author{
Leonardo Cabrer
}

\begin{abstract}
In this paper subvarieties of pseudocomplemented distributive lattices are classified by their unification type. We determine the unification type of every particular unification problem in each subvariety of pseudocomplemented distributive lattices.
\end{abstract}

\section{Introduction}

Syntactic unification theory is concerned with the problem of finding a substitution that equalizes a finite set of pairs of terms simultaneously. More precisely, given a set of function symbols $\mathscr{L}$ and a finite set of pairs of $\mathscr{L}$-terms $U=\left\{\left(t_{1}, s_{1}\right), \ldots,\left(t_{m}, s_{m}\right)\right\}$, called a unification problem, a unifier for $U$ is a substitution $\sigma$ defined on the set of variables of the terms in $U$ such that $\sigma\left(t_{i}\right)=\sigma\left(s_{i}\right)$ for each $i \in\{1, \ldots, m\}$. In many applications the operations in $\mathscr{L}$ are assumed to satisfy certain conditions that can be expressed by equations, such as associativity, commutativity, and idempotency. Then syntactic unification evolves into equational unification. Given an equational theory $E$ in the language $\mathscr{L}$, a unifier for $U$ is now asked to send the terms in each pair $\left(t_{i}, s_{i}\right) \in U$ to terms $\sigma\left(t_{i}\right)$ and $\sigma\left(s_{i}\right)$ that are equivalent for $E$ (in symbols, $\sigma\left(t_{i}\right) \approx_{E} \sigma\left(s_{i}\right)$ ).

Once a particular unification problem is known to admit $E$-unifiers, the next task is to find a complete description of its unifiers. For that we first observe that if $\sigma$ is an $E$-unifier for $U$, then $\gamma \circ \sigma$ is also an $E$-unifier for $U$, whenever $\gamma$ is a substitution such that $\gamma \circ \sigma$ is well defined. In this case, we say that $\sigma$ is more general than $\gamma \circ \sigma$. Therefore, a useful way to determine all the unifiers of a particular problem is to calculate a family of unifiers such that any other unifier of the problem is less general

Received June 11, 2012; accepted February 10, 2014

First published online September 12, 2016

2010 Mathematics Subject Classification: Primary 06D15; Secondary 08B30, 06D50, 03G10

Keywords: unification type, equational unification, projective algebra, pseudocomplemented lattice, topological duality

C 2016 by University of Notre Dame $\quad 10.1215 / 00294527-3659307$ 


\title{
Unification on Subvarieties of Pseudocomplemented Distributive Lattices
}

\author{
Leonardo Cabrer
}

\begin{abstract}
In this paper subvarieties of pseudocomplemented distributive lattices are classified by their unification type. We determine the unification type of every particular unification problem in each subvariety of pseudocomplemented distributive lattices.
\end{abstract}

\section{Introduction}

Syntactic unification theory is concerned with the problem of finding a substitution that equalizes a finite set of pairs of terms simultaneously. More precisely, given a set of function symbols $\mathscr{L}$ and a finite set of pairs of $\mathscr{L}$-terms $U=\left\{\left(t_{1}, s_{1}\right), \ldots,\left(t_{m}, s_{m}\right)\right\}$, called a unification problem, a unifier for $U$ is a substitution $\sigma$ defined on the set of variables of the terms in $U$ such that $\sigma\left(t_{i}\right)=\sigma\left(s_{i}\right)$ for each $i \in\{1, \ldots, m\}$. In many applications the operations in $\mathscr{L}$ are assumed to satisfy certain conditions that can be expressed by equations, such as associativity, commutativity, and idempotency. Then syntactic unification evolves into equational unification. Given an equational theory $E$ in the language $\mathscr{L}$, a unifier for $U$ is now asked to send the terms in each pair $\left(t_{i}, s_{i}\right) \in U$ to terms $\sigma\left(t_{i}\right)$ and $\sigma\left(s_{i}\right)$ that are equivalent for $E$ (in symbols, $\sigma\left(t_{i}\right) \approx_{E} \sigma\left(s_{i}\right)$ ).

Once a particular unification problem is known to admit $E$-unifiers, the next task is to find a complete description of its unifiers. For that we first observe that if $\sigma$ is an $E$-unifier for $U$, then $\gamma \circ \sigma$ is also an $E$-unifier for $U$, whenever $\gamma$ is a substitution such that $\gamma \circ \sigma$ is well defined. In this case, we say that $\sigma$ is more general than $\gamma \circ \sigma$. Therefore, a useful way to determine all the unifiers of a particular problem is to calculate a family of unifiers such that any other unifier of the problem is less general

Received June 11, 2012; accepted February 10, 2014

First published online September 12, 2016

2010 Mathematics Subject Classification: Primary 06D15; Secondary 08B30, 06D50, 03G10

Keywords: unification type, equational unification, projective algebra, pseudocomplemented lattice, topological duality

C 2016 by University of Notre Dame $\quad 10.1215 / 00294527-3659307$ 


\title{
Unification on Subvarieties of Pseudocomplemented Distributive Lattices
}

\author{
Leonardo Cabrer
}

\begin{abstract}
In this paper subvarieties of pseudocomplemented distributive lattices are classified by their unification type. We determine the unification type of every particular unification problem in each subvariety of pseudocomplemented distributive lattices.
\end{abstract}

\section{Introduction}

Syntactic unification theory is concerned with the problem of finding a substitution that equalizes a finite set of pairs of terms simultaneously. More precisely, given a set of function symbols $\mathscr{L}$ and a finite set of pairs of $\mathscr{L}$-terms $U=\left\{\left(t_{1}, s_{1}\right), \ldots,\left(t_{m}, s_{m}\right)\right\}$, called a unification problem, a unifier for $U$ is a substitution $\sigma$ defined on the set of variables of the terms in $U$ such that $\sigma\left(t_{i}\right)=\sigma\left(s_{i}\right)$ for each $i \in\{1, \ldots, m\}$. In many applications the operations in $\mathscr{L}$ are assumed to satisfy certain conditions that can be expressed by equations, such as associativity, commutativity, and idempotency. Then syntactic unification evolves into equational unification. Given an equational theory $E$ in the language $\mathscr{L}$, a unifier for $U$ is now asked to send the terms in each pair $\left(t_{i}, s_{i}\right) \in U$ to terms $\sigma\left(t_{i}\right)$ and $\sigma\left(s_{i}\right)$ that are equivalent for $E$ (in symbols, $\sigma\left(t_{i}\right) \approx_{E} \sigma\left(s_{i}\right)$ ).

Once a particular unification problem is known to admit $E$-unifiers, the next task is to find a complete description of its unifiers. For that we first observe that if $\sigma$ is an $E$-unifier for $U$, then $\gamma \circ \sigma$ is also an $E$-unifier for $U$, whenever $\gamma$ is a substitution such that $\gamma \circ \sigma$ is well defined. In this case, we say that $\sigma$ is more general than $\gamma \circ \sigma$. Therefore, a useful way to determine all the unifiers of a particular problem is to calculate a family of unifiers such that any other unifier of the problem is less general

Received June 11, 2012; accepted February 10, 2014

First published online September 12, 2016

2010 Mathematics Subject Classification: Primary 06D15; Secondary 08B30, 06D50, 03G10

Keywords: unification type, equational unification, projective algebra, pseudocomplemented lattice, topological duality

C 2016 by University of Notre Dame $\quad 10.1215 / 00294527-3659307$ 


\title{
Unification on Subvarieties of Pseudocomplemented Distributive Lattices
}

\author{
Leonardo Cabrer
}

\begin{abstract}
In this paper subvarieties of pseudocomplemented distributive lattices are classified by their unification type. We determine the unification type of every particular unification problem in each subvariety of pseudocomplemented distributive lattices.
\end{abstract}

\section{Introduction}

Syntactic unification theory is concerned with the problem of finding a substitution that equalizes a finite set of pairs of terms simultaneously. More precisely, given a set of function symbols $\mathscr{L}$ and a finite set of pairs of $\mathscr{L}$-terms $U=\left\{\left(t_{1}, s_{1}\right), \ldots,\left(t_{m}, s_{m}\right)\right\}$, called a unification problem, a unifier for $U$ is a substitution $\sigma$ defined on the set of variables of the terms in $U$ such that $\sigma\left(t_{i}\right)=\sigma\left(s_{i}\right)$ for each $i \in\{1, \ldots, m\}$. In many applications the operations in $\mathscr{L}$ are assumed to satisfy certain conditions that can be expressed by equations, such as associativity, commutativity, and idempotency. Then syntactic unification evolves into equational unification. Given an equational theory $E$ in the language $\mathscr{L}$, a unifier for $U$ is now asked to send the terms in each pair $\left(t_{i}, s_{i}\right) \in U$ to terms $\sigma\left(t_{i}\right)$ and $\sigma\left(s_{i}\right)$ that are equivalent for $E$ (in symbols, $\sigma\left(t_{i}\right) \approx_{E} \sigma\left(s_{i}\right)$ ).

Once a particular unification problem is known to admit $E$-unifiers, the next task is to find a complete description of its unifiers. For that we first observe that if $\sigma$ is an $E$-unifier for $U$, then $\gamma \circ \sigma$ is also an $E$-unifier for $U$, whenever $\gamma$ is a substitution such that $\gamma \circ \sigma$ is well defined. In this case, we say that $\sigma$ is more general than $\gamma \circ \sigma$. Therefore, a useful way to determine all the unifiers of a particular problem is to calculate a family of unifiers such that any other unifier of the problem is less general

Received June 11, 2012; accepted February 10, 2014

First published online September 12, 2016

2010 Mathematics Subject Classification: Primary 06D15; Secondary 08B30, 06D50, 03G10

Keywords: unification type, equational unification, projective algebra, pseudocomplemented lattice, topological duality

C 2016 by University of Notre Dame $\quad 10.1215 / 00294527-3659307$ 


\title{
Unification on Subvarieties of Pseudocomplemented Distributive Lattices
}

\author{
Leonardo Cabrer
}

\begin{abstract}
In this paper subvarieties of pseudocomplemented distributive lattices are classified by their unification type. We determine the unification type of every particular unification problem in each subvariety of pseudocomplemented distributive lattices.
\end{abstract}

\section{Introduction}

Syntactic unification theory is concerned with the problem of finding a substitution that equalizes a finite set of pairs of terms simultaneously. More precisely, given a set of function symbols $\mathscr{L}$ and a finite set of pairs of $\mathscr{L}$-terms $U=\left\{\left(t_{1}, s_{1}\right), \ldots,\left(t_{m}, s_{m}\right)\right\}$, called a unification problem, a unifier for $U$ is a substitution $\sigma$ defined on the set of variables of the terms in $U$ such that $\sigma\left(t_{i}\right)=\sigma\left(s_{i}\right)$ for each $i \in\{1, \ldots, m\}$. In many applications the operations in $\mathscr{L}$ are assumed to satisfy certain conditions that can be expressed by equations, such as associativity, commutativity, and idempotency. Then syntactic unification evolves into equational unification. Given an equational theory $E$ in the language $\mathscr{L}$, a unifier for $U$ is now asked to send the terms in each pair $\left(t_{i}, s_{i}\right) \in U$ to terms $\sigma\left(t_{i}\right)$ and $\sigma\left(s_{i}\right)$ that are equivalent for $E$ (in symbols, $\sigma\left(t_{i}\right) \approx_{E} \sigma\left(s_{i}\right)$ ).

Once a particular unification problem is known to admit $E$-unifiers, the next task is to find a complete description of its unifiers. For that we first observe that if $\sigma$ is an $E$-unifier for $U$, then $\gamma \circ \sigma$ is also an $E$-unifier for $U$, whenever $\gamma$ is a substitution such that $\gamma \circ \sigma$ is well defined. In this case, we say that $\sigma$ is more general than $\gamma \circ \sigma$. Therefore, a useful way to determine all the unifiers of a particular problem is to calculate a family of unifiers such that any other unifier of the problem is less general

Received June 11, 2012; accepted February 10, 2014

First published online September 12, 2016

2010 Mathematics Subject Classification: Primary 06D15; Secondary 08B30, 06D50, 03G10

Keywords: unification type, equational unification, projective algebra, pseudocomplemented lattice, topological duality

C 2016 by University of Notre Dame $\quad 10.1215 / 00294527-3659307$ 


\title{
Unification on Subvarieties of Pseudocomplemented Distributive Lattices
}

\author{
Leonardo Cabrer
}

\begin{abstract}
In this paper subvarieties of pseudocomplemented distributive lattices are classified by their unification type. We determine the unification type of every particular unification problem in each subvariety of pseudocomplemented distributive lattices.
\end{abstract}

\section{Introduction}

Syntactic unification theory is concerned with the problem of finding a substitution that equalizes a finite set of pairs of terms simultaneously. More precisely, given a set of function symbols $\mathscr{L}$ and a finite set of pairs of $\mathscr{L}$-terms $U=\left\{\left(t_{1}, s_{1}\right), \ldots,\left(t_{m}, s_{m}\right)\right\}$, called a unification problem, a unifier for $U$ is a substitution $\sigma$ defined on the set of variables of the terms in $U$ such that $\sigma\left(t_{i}\right)=\sigma\left(s_{i}\right)$ for each $i \in\{1, \ldots, m\}$. In many applications the operations in $\mathscr{L}$ are assumed to satisfy certain conditions that can be expressed by equations, such as associativity, commutativity, and idempotency. Then syntactic unification evolves into equational unification. Given an equational theory $E$ in the language $\mathscr{L}$, a unifier for $U$ is now asked to send the terms in each pair $\left(t_{i}, s_{i}\right) \in U$ to terms $\sigma\left(t_{i}\right)$ and $\sigma\left(s_{i}\right)$ that are equivalent for $E$ (in symbols, $\sigma\left(t_{i}\right) \approx_{E} \sigma\left(s_{i}\right)$ ).

Once a particular unification problem is known to admit $E$-unifiers, the next task is to find a complete description of its unifiers. For that we first observe that if $\sigma$ is an $E$-unifier for $U$, then $\gamma \circ \sigma$ is also an $E$-unifier for $U$, whenever $\gamma$ is a substitution such that $\gamma \circ \sigma$ is well defined. In this case, we say that $\sigma$ is more general than $\gamma \circ \sigma$. Therefore, a useful way to determine all the unifiers of a particular problem is to calculate a family of unifiers such that any other unifier of the problem is less general

Received June 11, 2012; accepted February 10, 2014

First published online September 12, 2016

2010 Mathematics Subject Classification: Primary 06D15; Secondary 08B30, 06D50, 03G10

Keywords: unification type, equational unification, projective algebra, pseudocomplemented lattice, topological duality

C 2016 by University of Notre Dame $\quad 10.1215 / 00294527-3659307$ 


\title{
Unification on Subvarieties of Pseudocomplemented Distributive Lattices
}

\author{
Leonardo Cabrer
}

\begin{abstract}
In this paper subvarieties of pseudocomplemented distributive lattices are classified by their unification type. We determine the unification type of every particular unification problem in each subvariety of pseudocomplemented distributive lattices.
\end{abstract}

\section{Introduction}

Syntactic unification theory is concerned with the problem of finding a substitution that equalizes a finite set of pairs of terms simultaneously. More precisely, given a set of function symbols $\mathscr{L}$ and a finite set of pairs of $\mathscr{L}$-terms $U=\left\{\left(t_{1}, s_{1}\right), \ldots,\left(t_{m}, s_{m}\right)\right\}$, called a unification problem, a unifier for $U$ is a substitution $\sigma$ defined on the set of variables of the terms in $U$ such that $\sigma\left(t_{i}\right)=\sigma\left(s_{i}\right)$ for each $i \in\{1, \ldots, m\}$. In many applications the operations in $\mathscr{L}$ are assumed to satisfy certain conditions that can be expressed by equations, such as associativity, commutativity, and idempotency. Then syntactic unification evolves into equational unification. Given an equational theory $E$ in the language $\mathscr{L}$, a unifier for $U$ is now asked to send the terms in each pair $\left(t_{i}, s_{i}\right) \in U$ to terms $\sigma\left(t_{i}\right)$ and $\sigma\left(s_{i}\right)$ that are equivalent for $E$ (in symbols, $\sigma\left(t_{i}\right) \approx_{E} \sigma\left(s_{i}\right)$ ).

Once a particular unification problem is known to admit $E$-unifiers, the next task is to find a complete description of its unifiers. For that we first observe that if $\sigma$ is an $E$-unifier for $U$, then $\gamma \circ \sigma$ is also an $E$-unifier for $U$, whenever $\gamma$ is a substitution such that $\gamma \circ \sigma$ is well defined. In this case, we say that $\sigma$ is more general than $\gamma \circ \sigma$. Therefore, a useful way to determine all the unifiers of a particular problem is to calculate a family of unifiers such that any other unifier of the problem is less general

Received June 11, 2012; accepted February 10, 2014

First published online September 12, 2016

2010 Mathematics Subject Classification: Primary 06D15; Secondary 08B30, 06D50, 03G10

Keywords: unification type, equational unification, projective algebra, pseudocomplemented lattice, topological duality

C 2016 by University of Notre Dame $\quad 10.1215 / 00294527-3659307$ 


\title{
Unification on Subvarieties of Pseudocomplemented Distributive Lattices
}

\author{
Leonardo Cabrer
}

\begin{abstract}
In this paper subvarieties of pseudocomplemented distributive lattices are classified by their unification type. We determine the unification type of every particular unification problem in each subvariety of pseudocomplemented distributive lattices.
\end{abstract}

\section{Introduction}

Syntactic unification theory is concerned with the problem of finding a substitution that equalizes a finite set of pairs of terms simultaneously. More precisely, given a set of function symbols $\mathscr{L}$ and a finite set of pairs of $\mathscr{L}$-terms $U=\left\{\left(t_{1}, s_{1}\right), \ldots,\left(t_{m}, s_{m}\right)\right\}$, called a unification problem, a unifier for $U$ is a substitution $\sigma$ defined on the set of variables of the terms in $U$ such that $\sigma\left(t_{i}\right)=\sigma\left(s_{i}\right)$ for each $i \in\{1, \ldots, m\}$. In many applications the operations in $\mathscr{L}$ are assumed to satisfy certain conditions that can be expressed by equations, such as associativity, commutativity, and idempotency. Then syntactic unification evolves into equational unification. Given an equational theory $E$ in the language $\mathscr{L}$, a unifier for $U$ is now asked to send the terms in each pair $\left(t_{i}, s_{i}\right) \in U$ to terms $\sigma\left(t_{i}\right)$ and $\sigma\left(s_{i}\right)$ that are equivalent for $E$ (in symbols, $\sigma\left(t_{i}\right) \approx_{E} \sigma\left(s_{i}\right)$ ).

Once a particular unification problem is known to admit $E$-unifiers, the next task is to find a complete description of its unifiers. For that we first observe that if $\sigma$ is an $E$-unifier for $U$, then $\gamma \circ \sigma$ is also an $E$-unifier for $U$, whenever $\gamma$ is a substitution such that $\gamma \circ \sigma$ is well defined. In this case, we say that $\sigma$ is more general than $\gamma \circ \sigma$. Therefore, a useful way to determine all the unifiers of a particular problem is to calculate a family of unifiers such that any other unifier of the problem is less general

Received June 11, 2012; accepted February 10, 2014

First published online September 12, 2016

2010 Mathematics Subject Classification: Primary 06D15; Secondary 08B30, 06D50, 03G10

Keywords: unification type, equational unification, projective algebra, pseudocomplemented lattice, topological duality

C 2016 by University of Notre Dame $\quad 10.1215 / 00294527-3659307$ 


\title{
Unification on Subvarieties of Pseudocomplemented Distributive Lattices
}

\author{
Leonardo Cabrer
}

\begin{abstract}
In this paper subvarieties of pseudocomplemented distributive lattices are classified by their unification type. We determine the unification type of every particular unification problem in each subvariety of pseudocomplemented distributive lattices.
\end{abstract}

\section{Introduction}

Syntactic unification theory is concerned with the problem of finding a substitution that equalizes a finite set of pairs of terms simultaneously. More precisely, given a set of function symbols $\mathscr{L}$ and a finite set of pairs of $\mathscr{L}$-terms $U=\left\{\left(t_{1}, s_{1}\right), \ldots,\left(t_{m}, s_{m}\right)\right\}$, called a unification problem, a unifier for $U$ is a substitution $\sigma$ defined on the set of variables of the terms in $U$ such that $\sigma\left(t_{i}\right)=\sigma\left(s_{i}\right)$ for each $i \in\{1, \ldots, m\}$. In many applications the operations in $\mathscr{L}$ are assumed to satisfy certain conditions that can be expressed by equations, such as associativity, commutativity, and idempotency. Then syntactic unification evolves into equational unification. Given an equational theory $E$ in the language $\mathscr{L}$, a unifier for $U$ is now asked to send the terms in each pair $\left(t_{i}, s_{i}\right) \in U$ to terms $\sigma\left(t_{i}\right)$ and $\sigma\left(s_{i}\right)$ that are equivalent for $E$ (in symbols, $\sigma\left(t_{i}\right) \approx_{E} \sigma\left(s_{i}\right)$ ).

Once a particular unification problem is known to admit $E$-unifiers, the next task is to find a complete description of its unifiers. For that we first observe that if $\sigma$ is an $E$-unifier for $U$, then $\gamma \circ \sigma$ is also an $E$-unifier for $U$, whenever $\gamma$ is a substitution such that $\gamma \circ \sigma$ is well defined. In this case, we say that $\sigma$ is more general than $\gamma \circ \sigma$. Therefore, a useful way to determine all the unifiers of a particular problem is to calculate a family of unifiers such that any other unifier of the problem is less general

Received June 11, 2012; accepted February 10, 2014

First published online September 12, 2016

2010 Mathematics Subject Classification: Primary 06D15; Secondary 08B30, 06D50, 03G10

Keywords: unification type, equational unification, projective algebra, pseudocomplemented lattice, topological duality

C 2016 by University of Notre Dame $\quad 10.1215 / 00294527-3659307$ 


\title{
Unification on Subvarieties of Pseudocomplemented Distributive Lattices
}

\author{
Leonardo Cabrer
}

\begin{abstract}
In this paper subvarieties of pseudocomplemented distributive lattices are classified by their unification type. We determine the unification type of every particular unification problem in each subvariety of pseudocomplemented distributive lattices.
\end{abstract}

\section{Introduction}

Syntactic unification theory is concerned with the problem of finding a substitution that equalizes a finite set of pairs of terms simultaneously. More precisely, given a set of function symbols $\mathscr{L}$ and a finite set of pairs of $\mathscr{L}$-terms $U=\left\{\left(t_{1}, s_{1}\right), \ldots,\left(t_{m}, s_{m}\right)\right\}$, called a unification problem, a unifier for $U$ is a substitution $\sigma$ defined on the set of variables of the terms in $U$ such that $\sigma\left(t_{i}\right)=\sigma\left(s_{i}\right)$ for each $i \in\{1, \ldots, m\}$. In many applications the operations in $\mathscr{L}$ are assumed to satisfy certain conditions that can be expressed by equations, such as associativity, commutativity, and idempotency. Then syntactic unification evolves into equational unification. Given an equational theory $E$ in the language $\mathscr{L}$, a unifier for $U$ is now asked to send the terms in each pair $\left(t_{i}, s_{i}\right) \in U$ to terms $\sigma\left(t_{i}\right)$ and $\sigma\left(s_{i}\right)$ that are equivalent for $E$ (in symbols, $\sigma\left(t_{i}\right) \approx_{E} \sigma\left(s_{i}\right)$ ).

Once a particular unification problem is known to admit $E$-unifiers, the next task is to find a complete description of its unifiers. For that we first observe that if $\sigma$ is an $E$-unifier for $U$, then $\gamma \circ \sigma$ is also an $E$-unifier for $U$, whenever $\gamma$ is a substitution such that $\gamma \circ \sigma$ is well defined. In this case, we say that $\sigma$ is more general than $\gamma \circ \sigma$. Therefore, a useful way to determine all the unifiers of a particular problem is to calculate a family of unifiers such that any other unifier of the problem is less general

Received June 11, 2012; accepted February 10, 2014

First published online September 12, 2016

2010 Mathematics Subject Classification: Primary 06D15; Secondary 08B30, 06D50, 03G10

Keywords: unification type, equational unification, projective algebra, pseudocomplemented lattice, topological duality

C 2016 by University of Notre Dame $\quad 10.1215 / 00294527-3659307$ 


\title{
Unification on Subvarieties of Pseudocomplemented Distributive Lattices
}

\author{
Leonardo Cabrer
}

\begin{abstract}
In this paper subvarieties of pseudocomplemented distributive lattices are classified by their unification type. We determine the unification type of every particular unification problem in each subvariety of pseudocomplemented distributive lattices.
\end{abstract}

\section{Introduction}

Syntactic unification theory is concerned with the problem of finding a substitution that equalizes a finite set of pairs of terms simultaneously. More precisely, given a set of function symbols $\mathscr{L}$ and a finite set of pairs of $\mathscr{L}$-terms $U=\left\{\left(t_{1}, s_{1}\right), \ldots,\left(t_{m}, s_{m}\right)\right\}$, called a unification problem, a unifier for $U$ is a substitution $\sigma$ defined on the set of variables of the terms in $U$ such that $\sigma\left(t_{i}\right)=\sigma\left(s_{i}\right)$ for each $i \in\{1, \ldots, m\}$. In many applications the operations in $\mathscr{L}$ are assumed to satisfy certain conditions that can be expressed by equations, such as associativity, commutativity, and idempotency. Then syntactic unification evolves into equational unification. Given an equational theory $E$ in the language $\mathscr{L}$, a unifier for $U$ is now asked to send the terms in each pair $\left(t_{i}, s_{i}\right) \in U$ to terms $\sigma\left(t_{i}\right)$ and $\sigma\left(s_{i}\right)$ that are equivalent for $E$ (in symbols, $\sigma\left(t_{i}\right) \approx_{E} \sigma\left(s_{i}\right)$ ).

Once a particular unification problem is known to admit $E$-unifiers, the next task is to find a complete description of its unifiers. For that we first observe that if $\sigma$ is an $E$-unifier for $U$, then $\gamma \circ \sigma$ is also an $E$-unifier for $U$, whenever $\gamma$ is a substitution such that $\gamma \circ \sigma$ is well defined. In this case, we say that $\sigma$ is more general than $\gamma \circ \sigma$. Therefore, a useful way to determine all the unifiers of a particular problem is to calculate a family of unifiers such that any other unifier of the problem is less general

Received June 11, 2012; accepted February 10, 2014

First published online September 12, 2016

2010 Mathematics Subject Classification: Primary 06D15; Secondary 08B30, 06D50, 03G10

Keywords: unification type, equational unification, projective algebra, pseudocomplemented lattice, topological duality

C 2016 by University of Notre Dame $\quad 10.1215 / 00294527-3659307$ 


\title{
Unification on Subvarieties of Pseudocomplemented Distributive Lattices
}

\author{
Leonardo Cabrer
}

\begin{abstract}
In this paper subvarieties of pseudocomplemented distributive lattices are classified by their unification type. We determine the unification type of every particular unification problem in each subvariety of pseudocomplemented distributive lattices.
\end{abstract}

\section{Introduction}

Syntactic unification theory is concerned with the problem of finding a substitution that equalizes a finite set of pairs of terms simultaneously. More precisely, given a set of function symbols $\mathscr{L}$ and a finite set of pairs of $\mathscr{L}$-terms $U=\left\{\left(t_{1}, s_{1}\right), \ldots,\left(t_{m}, s_{m}\right)\right\}$, called a unification problem, a unifier for $U$ is a substitution $\sigma$ defined on the set of variables of the terms in $U$ such that $\sigma\left(t_{i}\right)=\sigma\left(s_{i}\right)$ for each $i \in\{1, \ldots, m\}$. In many applications the operations in $\mathscr{L}$ are assumed to satisfy certain conditions that can be expressed by equations, such as associativity, commutativity, and idempotency. Then syntactic unification evolves into equational unification. Given an equational theory $E$ in the language $\mathscr{L}$, a unifier for $U$ is now asked to send the terms in each pair $\left(t_{i}, s_{i}\right) \in U$ to terms $\sigma\left(t_{i}\right)$ and $\sigma\left(s_{i}\right)$ that are equivalent for $E$ (in symbols, $\sigma\left(t_{i}\right) \approx_{E} \sigma\left(s_{i}\right)$ ).

Once a particular unification problem is known to admit $E$-unifiers, the next task is to find a complete description of its unifiers. For that we first observe that if $\sigma$ is an $E$-unifier for $U$, then $\gamma \circ \sigma$ is also an $E$-unifier for $U$, whenever $\gamma$ is a substitution such that $\gamma \circ \sigma$ is well defined. In this case, we say that $\sigma$ is more general than $\gamma \circ \sigma$. Therefore, a useful way to determine all the unifiers of a particular problem is to calculate a family of unifiers such that any other unifier of the problem is less general

Received June 11, 2012; accepted February 10, 2014

First published online September 12, 2016

2010 Mathematics Subject Classification: Primary 06D15; Secondary 08B30, 06D50, 03G10

Keywords: unification type, equational unification, projective algebra, pseudocomplemented lattice, topological duality

C 2016 by University of Notre Dame $\quad 10.1215 / 00294527-3659307$ 


\title{
Unification on Subvarieties of Pseudocomplemented Distributive Lattices
}

\author{
Leonardo Cabrer
}

\begin{abstract}
In this paper subvarieties of pseudocomplemented distributive lattices are classified by their unification type. We determine the unification type of every particular unification problem in each subvariety of pseudocomplemented distributive lattices.
\end{abstract}

\section{Introduction}

Syntactic unification theory is concerned with the problem of finding a substitution that equalizes a finite set of pairs of terms simultaneously. More precisely, given a set of function symbols $\mathscr{L}$ and a finite set of pairs of $\mathscr{L}$-terms $U=\left\{\left(t_{1}, s_{1}\right), \ldots,\left(t_{m}, s_{m}\right)\right\}$, called a unification problem, a unifier for $U$ is a substitution $\sigma$ defined on the set of variables of the terms in $U$ such that $\sigma\left(t_{i}\right)=\sigma\left(s_{i}\right)$ for each $i \in\{1, \ldots, m\}$. In many applications the operations in $\mathscr{L}$ are assumed to satisfy certain conditions that can be expressed by equations, such as associativity, commutativity, and idempotency. Then syntactic unification evolves into equational unification. Given an equational theory $E$ in the language $\mathscr{L}$, a unifier for $U$ is now asked to send the terms in each pair $\left(t_{i}, s_{i}\right) \in U$ to terms $\sigma\left(t_{i}\right)$ and $\sigma\left(s_{i}\right)$ that are equivalent for $E$ (in symbols, $\sigma\left(t_{i}\right) \approx_{E} \sigma\left(s_{i}\right)$ ).

Once a particular unification problem is known to admit $E$-unifiers, the next task is to find a complete description of its unifiers. For that we first observe that if $\sigma$ is an $E$-unifier for $U$, then $\gamma \circ \sigma$ is also an $E$-unifier for $U$, whenever $\gamma$ is a substitution such that $\gamma \circ \sigma$ is well defined. In this case, we say that $\sigma$ is more general than $\gamma \circ \sigma$. Therefore, a useful way to determine all the unifiers of a particular problem is to calculate a family of unifiers such that any other unifier of the problem is less general

Received June 11, 2012; accepted February 10, 2014

First published online September 12, 2016

2010 Mathematics Subject Classification: Primary 06D15; Secondary 08B30, 06D50, 03G10

Keywords: unification type, equational unification, projective algebra, pseudocomplemented lattice, topological duality

C 2016 by University of Notre Dame $\quad 10.1215 / 00294527-3659307$ 


\title{
Unification on Subvarieties of Pseudocomplemented Distributive Lattices
}

\author{
Leonardo Cabrer
}

\begin{abstract}
In this paper subvarieties of pseudocomplemented distributive lattices are classified by their unification type. We determine the unification type of every particular unification problem in each subvariety of pseudocomplemented distributive lattices.
\end{abstract}

\section{Introduction}

Syntactic unification theory is concerned with the problem of finding a substitution that equalizes a finite set of pairs of terms simultaneously. More precisely, given a set of function symbols $\mathscr{L}$ and a finite set of pairs of $\mathscr{L}$-terms $U=\left\{\left(t_{1}, s_{1}\right), \ldots,\left(t_{m}, s_{m}\right)\right\}$, called a unification problem, a unifier for $U$ is a substitution $\sigma$ defined on the set of variables of the terms in $U$ such that $\sigma\left(t_{i}\right)=\sigma\left(s_{i}\right)$ for each $i \in\{1, \ldots, m\}$. In many applications the operations in $\mathscr{L}$ are assumed to satisfy certain conditions that can be expressed by equations, such as associativity, commutativity, and idempotency. Then syntactic unification evolves into equational unification. Given an equational theory $E$ in the language $\mathscr{L}$, a unifier for $U$ is now asked to send the terms in each pair $\left(t_{i}, s_{i}\right) \in U$ to terms $\sigma\left(t_{i}\right)$ and $\sigma\left(s_{i}\right)$ that are equivalent for $E$ (in symbols, $\sigma\left(t_{i}\right) \approx_{E} \sigma\left(s_{i}\right)$ ).

Once a particular unification problem is known to admit $E$-unifiers, the next task is to find a complete description of its unifiers. For that we first observe that if $\sigma$ is an $E$-unifier for $U$, then $\gamma \circ \sigma$ is also an $E$-unifier for $U$, whenever $\gamma$ is a substitution such that $\gamma \circ \sigma$ is well defined. In this case, we say that $\sigma$ is more general than $\gamma \circ \sigma$. Therefore, a useful way to determine all the unifiers of a particular problem is to calculate a family of unifiers such that any other unifier of the problem is less general

Received June 11, 2012; accepted February 10, 2014

First published online September 12, 2016

2010 Mathematics Subject Classification: Primary 06D15; Secondary 08B30, 06D50, 03G10

Keywords: unification type, equational unification, projective algebra, pseudocomplemented lattice, topological duality

C 2016 by University of Notre Dame $\quad 10.1215 / 00294527-3659307$ 


\title{
Unification on Subvarieties of Pseudocomplemented Distributive Lattices
}

\author{
Leonardo Cabrer
}

\begin{abstract}
In this paper subvarieties of pseudocomplemented distributive lattices are classified by their unification type. We determine the unification type of every particular unification problem in each subvariety of pseudocomplemented distributive lattices.
\end{abstract}

\section{Introduction}

Syntactic unification theory is concerned with the problem of finding a substitution that equalizes a finite set of pairs of terms simultaneously. More precisely, given a set of function symbols $\mathscr{L}$ and a finite set of pairs of $\mathscr{L}$-terms $U=\left\{\left(t_{1}, s_{1}\right), \ldots,\left(t_{m}, s_{m}\right)\right\}$, called a unification problem, a unifier for $U$ is a substitution $\sigma$ defined on the set of variables of the terms in $U$ such that $\sigma\left(t_{i}\right)=\sigma\left(s_{i}\right)$ for each $i \in\{1, \ldots, m\}$. In many applications the operations in $\mathscr{L}$ are assumed to satisfy certain conditions that can be expressed by equations, such as associativity, commutativity, and idempotency. Then syntactic unification evolves into equational unification. Given an equational theory $E$ in the language $\mathscr{L}$, a unifier for $U$ is now asked to send the terms in each pair $\left(t_{i}, s_{i}\right) \in U$ to terms $\sigma\left(t_{i}\right)$ and $\sigma\left(s_{i}\right)$ that are equivalent for $E$ (in symbols, $\sigma\left(t_{i}\right) \approx_{E} \sigma\left(s_{i}\right)$ ).

Once a particular unification problem is known to admit $E$-unifiers, the next task is to find a complete description of its unifiers. For that we first observe that if $\sigma$ is an $E$-unifier for $U$, then $\gamma \circ \sigma$ is also an $E$-unifier for $U$, whenever $\gamma$ is a substitution such that $\gamma \circ \sigma$ is well defined. In this case, we say that $\sigma$ is more general than $\gamma \circ \sigma$. Therefore, a useful way to determine all the unifiers of a particular problem is to calculate a family of unifiers such that any other unifier of the problem is less general

Received June 11, 2012; accepted February 10, 2014

First published online September 12, 2016

2010 Mathematics Subject Classification: Primary 06D15; Secondary 08B30, 06D50, 03G10

Keywords: unification type, equational unification, projective algebra, pseudocomplemented lattice, topological duality

C 2016 by University of Notre Dame $\quad 10.1215 / 00294527-3659307$ 


\title{
Unification on Subvarieties of Pseudocomplemented Distributive Lattices
}

\author{
Leonardo Cabrer
}

\begin{abstract}
In this paper subvarieties of pseudocomplemented distributive lattices are classified by their unification type. We determine the unification type of every particular unification problem in each subvariety of pseudocomplemented distributive lattices.
\end{abstract}

\section{Introduction}

Syntactic unification theory is concerned with the problem of finding a substitution that equalizes a finite set of pairs of terms simultaneously. More precisely, given a set of function symbols $\mathscr{L}$ and a finite set of pairs of $\mathscr{L}$-terms $U=\left\{\left(t_{1}, s_{1}\right), \ldots,\left(t_{m}, s_{m}\right)\right\}$, called a unification problem, a unifier for $U$ is a substitution $\sigma$ defined on the set of variables of the terms in $U$ such that $\sigma\left(t_{i}\right)=\sigma\left(s_{i}\right)$ for each $i \in\{1, \ldots, m\}$. In many applications the operations in $\mathscr{L}$ are assumed to satisfy certain conditions that can be expressed by equations, such as associativity, commutativity, and idempotency. Then syntactic unification evolves into equational unification. Given an equational theory $E$ in the language $\mathscr{L}$, a unifier for $U$ is now asked to send the terms in each pair $\left(t_{i}, s_{i}\right) \in U$ to terms $\sigma\left(t_{i}\right)$ and $\sigma\left(s_{i}\right)$ that are equivalent for $E$ (in symbols, $\sigma\left(t_{i}\right) \approx_{E} \sigma\left(s_{i}\right)$ ).

Once a particular unification problem is known to admit $E$-unifiers, the next task is to find a complete description of its unifiers. For that we first observe that if $\sigma$ is an $E$-unifier for $U$, then $\gamma \circ \sigma$ is also an $E$-unifier for $U$, whenever $\gamma$ is a substitution such that $\gamma \circ \sigma$ is well defined. In this case, we say that $\sigma$ is more general than $\gamma \circ \sigma$. Therefore, a useful way to determine all the unifiers of a particular problem is to calculate a family of unifiers such that any other unifier of the problem is less general

Received June 11, 2012; accepted February 10, 2014

First published online September 12, 2016

2010 Mathematics Subject Classification: Primary 06D15; Secondary 08B30, 06D50, 03G10

Keywords: unification type, equational unification, projective algebra, pseudocomplemented lattice, topological duality

C 2016 by University of Notre Dame $\quad 10.1215 / 00294527-3659307$ 


\title{
Unification on Subvarieties of Pseudocomplemented Distributive Lattices
}

\author{
Leonardo Cabrer
}

\begin{abstract}
In this paper subvarieties of pseudocomplemented distributive lattices are classified by their unification type. We determine the unification type of every particular unification problem in each subvariety of pseudocomplemented distributive lattices.
\end{abstract}

\section{Introduction}

Syntactic unification theory is concerned with the problem of finding a substitution that equalizes a finite set of pairs of terms simultaneously. More precisely, given a set of function symbols $\mathscr{L}$ and a finite set of pairs of $\mathscr{L}$-terms $U=\left\{\left(t_{1}, s_{1}\right), \ldots,\left(t_{m}, s_{m}\right)\right\}$, called a unification problem, a unifier for $U$ is a substitution $\sigma$ defined on the set of variables of the terms in $U$ such that $\sigma\left(t_{i}\right)=\sigma\left(s_{i}\right)$ for each $i \in\{1, \ldots, m\}$. In many applications the operations in $\mathscr{L}$ are assumed to satisfy certain conditions that can be expressed by equations, such as associativity, commutativity, and idempotency. Then syntactic unification evolves into equational unification. Given an equational theory $E$ in the language $\mathscr{L}$, a unifier for $U$ is now asked to send the terms in each pair $\left(t_{i}, s_{i}\right) \in U$ to terms $\sigma\left(t_{i}\right)$ and $\sigma\left(s_{i}\right)$ that are equivalent for $E$ (in symbols, $\sigma\left(t_{i}\right) \approx_{E} \sigma\left(s_{i}\right)$ ).

Once a particular unification problem is known to admit $E$-unifiers, the next task is to find a complete description of its unifiers. For that we first observe that if $\sigma$ is an $E$-unifier for $U$, then $\gamma \circ \sigma$ is also an $E$-unifier for $U$, whenever $\gamma$ is a substitution such that $\gamma \circ \sigma$ is well defined. In this case, we say that $\sigma$ is more general than $\gamma \circ \sigma$. Therefore, a useful way to determine all the unifiers of a particular problem is to calculate a family of unifiers such that any other unifier of the problem is less general

Received June 11, 2012; accepted February 10, 2014

First published online September 12, 2016

2010 Mathematics Subject Classification: Primary 06D15; Secondary 08B30, 06D50, 03G10

Keywords: unification type, equational unification, projective algebra, pseudocomplemented lattice, topological duality

C 2016 by University of Notre Dame $\quad 10.1215 / 00294527-3659307$ 


\title{
Unification on Subvarieties of Pseudocomplemented Distributive Lattices
}

\author{
Leonardo Cabrer
}

\begin{abstract}
In this paper subvarieties of pseudocomplemented distributive lattices are classified by their unification type. We determine the unification type of every particular unification problem in each subvariety of pseudocomplemented distributive lattices.
\end{abstract}

\section{Introduction}

Syntactic unification theory is concerned with the problem of finding a substitution that equalizes a finite set of pairs of terms simultaneously. More precisely, given a set of function symbols $\mathscr{L}$ and a finite set of pairs of $\mathscr{L}$-terms $U=\left\{\left(t_{1}, s_{1}\right), \ldots,\left(t_{m}, s_{m}\right)\right\}$, called a unification problem, a unifier for $U$ is a substitution $\sigma$ defined on the set of variables of the terms in $U$ such that $\sigma\left(t_{i}\right)=\sigma\left(s_{i}\right)$ for each $i \in\{1, \ldots, m\}$. In many applications the operations in $\mathscr{L}$ are assumed to satisfy certain conditions that can be expressed by equations, such as associativity, commutativity, and idempotency. Then syntactic unification evolves into equational unification. Given an equational theory $E$ in the language $\mathscr{L}$, a unifier for $U$ is now asked to send the terms in each pair $\left(t_{i}, s_{i}\right) \in U$ to terms $\sigma\left(t_{i}\right)$ and $\sigma\left(s_{i}\right)$ that are equivalent for $E$ (in symbols, $\sigma\left(t_{i}\right) \approx_{E} \sigma\left(s_{i}\right)$ ).

Once a particular unification problem is known to admit $E$-unifiers, the next task is to find a complete description of its unifiers. For that we first observe that if $\sigma$ is an $E$-unifier for $U$, then $\gamma \circ \sigma$ is also an $E$-unifier for $U$, whenever $\gamma$ is a substitution such that $\gamma \circ \sigma$ is well defined. In this case, we say that $\sigma$ is more general than $\gamma \circ \sigma$. Therefore, a useful way to determine all the unifiers of a particular problem is to calculate a family of unifiers such that any other unifier of the problem is less general

Received June 11, 2012; accepted February 10, 2014

First published online September 12, 2016

2010 Mathematics Subject Classification: Primary 06D15; Secondary 08B30, 06D50, 03G10

Keywords: unification type, equational unification, projective algebra, pseudocomplemented lattice, topological duality

C 2016 by University of Notre Dame $\quad 10.1215 / 00294527-3659307$ 


\title{
Unification on Subvarieties of Pseudocomplemented Distributive Lattices
}

\author{
Leonardo Cabrer
}

\begin{abstract}
In this paper subvarieties of pseudocomplemented distributive lattices are classified by their unification type. We determine the unification type of every particular unification problem in each subvariety of pseudocomplemented distributive lattices.
\end{abstract}

\section{Introduction}

Syntactic unification theory is concerned with the problem of finding a substitution that equalizes a finite set of pairs of terms simultaneously. More precisely, given a set of function symbols $\mathscr{L}$ and a finite set of pairs of $\mathscr{L}$-terms $U=\left\{\left(t_{1}, s_{1}\right), \ldots,\left(t_{m}, s_{m}\right)\right\}$, called a unification problem, a unifier for $U$ is a substitution $\sigma$ defined on the set of variables of the terms in $U$ such that $\sigma\left(t_{i}\right)=\sigma\left(s_{i}\right)$ for each $i \in\{1, \ldots, m\}$. In many applications the operations in $\mathscr{L}$ are assumed to satisfy certain conditions that can be expressed by equations, such as associativity, commutativity, and idempotency. Then syntactic unification evolves into equational unification. Given an equational theory $E$ in the language $\mathscr{L}$, a unifier for $U$ is now asked to send the terms in each pair $\left(t_{i}, s_{i}\right) \in U$ to terms $\sigma\left(t_{i}\right)$ and $\sigma\left(s_{i}\right)$ that are equivalent for $E$ (in symbols, $\sigma\left(t_{i}\right) \approx_{E} \sigma\left(s_{i}\right)$ ).

Once a particular unification problem is known to admit $E$-unifiers, the next task is to find a complete description of its unifiers. For that we first observe that if $\sigma$ is an $E$-unifier for $U$, then $\gamma \circ \sigma$ is also an $E$-unifier for $U$, whenever $\gamma$ is a substitution such that $\gamma \circ \sigma$ is well defined. In this case, we say that $\sigma$ is more general than $\gamma \circ \sigma$. Therefore, a useful way to determine all the unifiers of a particular problem is to calculate a family of unifiers such that any other unifier of the problem is less general

Received June 11, 2012; accepted February 10, 2014

First published online September 12, 2016

2010 Mathematics Subject Classification: Primary 06D15; Secondary 08B30, 06D50, 03G10

Keywords: unification type, equational unification, projective algebra, pseudocomplemented lattice, topological duality

C 2016 by University of Notre Dame $\quad 10.1215 / 00294527-3659307$ 


\title{
Unification on Subvarieties of Pseudocomplemented Distributive Lattices
}

\author{
Leonardo Cabrer
}

\begin{abstract}
In this paper subvarieties of pseudocomplemented distributive lattices are classified by their unification type. We determine the unification type of every particular unification problem in each subvariety of pseudocomplemented distributive lattices.
\end{abstract}

\section{Introduction}

Syntactic unification theory is concerned with the problem of finding a substitution that equalizes a finite set of pairs of terms simultaneously. More precisely, given a set of function symbols $\mathscr{L}$ and a finite set of pairs of $\mathscr{L}$-terms $U=\left\{\left(t_{1}, s_{1}\right), \ldots,\left(t_{m}, s_{m}\right)\right\}$, called a unification problem, a unifier for $U$ is a substitution $\sigma$ defined on the set of variables of the terms in $U$ such that $\sigma\left(t_{i}\right)=\sigma\left(s_{i}\right)$ for each $i \in\{1, \ldots, m\}$. In many applications the operations in $\mathscr{L}$ are assumed to satisfy certain conditions that can be expressed by equations, such as associativity, commutativity, and idempotency. Then syntactic unification evolves into equational unification. Given an equational theory $E$ in the language $\mathscr{L}$, a unifier for $U$ is now asked to send the terms in each pair $\left(t_{i}, s_{i}\right) \in U$ to terms $\sigma\left(t_{i}\right)$ and $\sigma\left(s_{i}\right)$ that are equivalent for $E$ (in symbols, $\sigma\left(t_{i}\right) \approx_{E} \sigma\left(s_{i}\right)$ ).

Once a particular unification problem is known to admit $E$-unifiers, the next task is to find a complete description of its unifiers. For that we first observe that if $\sigma$ is an $E$-unifier for $U$, then $\gamma \circ \sigma$ is also an $E$-unifier for $U$, whenever $\gamma$ is a substitution such that $\gamma \circ \sigma$ is well defined. In this case, we say that $\sigma$ is more general than $\gamma \circ \sigma$. Therefore, a useful way to determine all the unifiers of a particular problem is to calculate a family of unifiers such that any other unifier of the problem is less general

Received June 11, 2012; accepted February 10, 2014

First published online September 12, 2016

2010 Mathematics Subject Classification: Primary 06D15; Secondary 08B30, 06D50, 03G10

Keywords: unification type, equational unification, projective algebra, pseudocomplemented lattice, topological duality

C 2016 by University of Notre Dame $\quad 10.1215 / 00294527-3659307$ 


\title{
Unification on Subvarieties of Pseudocomplemented Distributive Lattices
}

\author{
Leonardo Cabrer
}

\begin{abstract}
In this paper subvarieties of pseudocomplemented distributive lattices are classified by their unification type. We determine the unification type of every particular unification problem in each subvariety of pseudocomplemented distributive lattices.
\end{abstract}

\section{Introduction}

Syntactic unification theory is concerned with the problem of finding a substitution that equalizes a finite set of pairs of terms simultaneously. More precisely, given a set of function symbols $\mathscr{L}$ and a finite set of pairs of $\mathscr{L}$-terms $U=\left\{\left(t_{1}, s_{1}\right), \ldots,\left(t_{m}, s_{m}\right)\right\}$, called a unification problem, a unifier for $U$ is a substitution $\sigma$ defined on the set of variables of the terms in $U$ such that $\sigma\left(t_{i}\right)=\sigma\left(s_{i}\right)$ for each $i \in\{1, \ldots, m\}$. In many applications the operations in $\mathscr{L}$ are assumed to satisfy certain conditions that can be expressed by equations, such as associativity, commutativity, and idempotency. Then syntactic unification evolves into equational unification. Given an equational theory $E$ in the language $\mathscr{L}$, a unifier for $U$ is now asked to send the terms in each pair $\left(t_{i}, s_{i}\right) \in U$ to terms $\sigma\left(t_{i}\right)$ and $\sigma\left(s_{i}\right)$ that are equivalent for $E$ (in symbols, $\sigma\left(t_{i}\right) \approx_{E} \sigma\left(s_{i}\right)$ ).

Once a particular unification problem is known to admit $E$-unifiers, the next task is to find a complete description of its unifiers. For that we first observe that if $\sigma$ is an $E$-unifier for $U$, then $\gamma \circ \sigma$ is also an $E$-unifier for $U$, whenever $\gamma$ is a substitution such that $\gamma \circ \sigma$ is well defined. In this case, we say that $\sigma$ is more general than $\gamma \circ \sigma$. Therefore, a useful way to determine all the unifiers of a particular problem is to calculate a family of unifiers such that any other unifier of the problem is less general

Received June 11, 2012; accepted February 10, 2014

First published online September 12, 2016

2010 Mathematics Subject Classification: Primary 06D15; Secondary 08B30, 06D50, 03G10

Keywords: unification type, equational unification, projective algebra, pseudocomplemented lattice, topological duality

C 2016 by University of Notre Dame $\quad 10.1215 / 00294527-3659307$ 


\title{
Unification on Subvarieties of Pseudocomplemented Distributive Lattices
}

\author{
Leonardo Cabrer
}

\begin{abstract}
In this paper subvarieties of pseudocomplemented distributive lattices are classified by their unification type. We determine the unification type of every particular unification problem in each subvariety of pseudocomplemented distributive lattices.
\end{abstract}

\section{Introduction}

Syntactic unification theory is concerned with the problem of finding a substitution that equalizes a finite set of pairs of terms simultaneously. More precisely, given a set of function symbols $\mathscr{L}$ and a finite set of pairs of $\mathscr{L}$-terms $U=\left\{\left(t_{1}, s_{1}\right), \ldots,\left(t_{m}, s_{m}\right)\right\}$, called a unification problem, a unifier for $U$ is a substitution $\sigma$ defined on the set of variables of the terms in $U$ such that $\sigma\left(t_{i}\right)=\sigma\left(s_{i}\right)$ for each $i \in\{1, \ldots, m\}$. In many applications the operations in $\mathscr{L}$ are assumed to satisfy certain conditions that can be expressed by equations, such as associativity, commutativity, and idempotency. Then syntactic unification evolves into equational unification. Given an equational theory $E$ in the language $\mathscr{L}$, a unifier for $U$ is now asked to send the terms in each pair $\left(t_{i}, s_{i}\right) \in U$ to terms $\sigma\left(t_{i}\right)$ and $\sigma\left(s_{i}\right)$ that are equivalent for $E$ (in symbols, $\sigma\left(t_{i}\right) \approx_{E} \sigma\left(s_{i}\right)$ ).

Once a particular unification problem is known to admit $E$-unifiers, the next task is to find a complete description of its unifiers. For that we first observe that if $\sigma$ is an $E$-unifier for $U$, then $\gamma \circ \sigma$ is also an $E$-unifier for $U$, whenever $\gamma$ is a substitution such that $\gamma \circ \sigma$ is well defined. In this case, we say that $\sigma$ is more general than $\gamma \circ \sigma$. Therefore, a useful way to determine all the unifiers of a particular problem is to calculate a family of unifiers such that any other unifier of the problem is less general

Received June 11, 2012; accepted February 10, 2014

First published online September 12, 2016

2010 Mathematics Subject Classification: Primary 06D15; Secondary 08B30, 06D50, 03G10

Keywords: unification type, equational unification, projective algebra, pseudocomplemented lattice, topological duality

C 2016 by University of Notre Dame $\quad 10.1215 / 00294527-3659307$ 


\title{
Unification on Subvarieties of Pseudocomplemented Distributive Lattices
}

\author{
Leonardo Cabrer
}

\begin{abstract}
In this paper subvarieties of pseudocomplemented distributive lattices are classified by their unification type. We determine the unification type of every particular unification problem in each subvariety of pseudocomplemented distributive lattices.
\end{abstract}

\section{Introduction}

Syntactic unification theory is concerned with the problem of finding a substitution that equalizes a finite set of pairs of terms simultaneously. More precisely, given a set of function symbols $\mathscr{L}$ and a finite set of pairs of $\mathscr{L}$-terms $U=\left\{\left(t_{1}, s_{1}\right), \ldots,\left(t_{m}, s_{m}\right)\right\}$, called a unification problem, a unifier for $U$ is a substitution $\sigma$ defined on the set of variables of the terms in $U$ such that $\sigma\left(t_{i}\right)=\sigma\left(s_{i}\right)$ for each $i \in\{1, \ldots, m\}$. In many applications the operations in $\mathscr{L}$ are assumed to satisfy certain conditions that can be expressed by equations, such as associativity, commutativity, and idempotency. Then syntactic unification evolves into equational unification. Given an equational theory $E$ in the language $\mathscr{L}$, a unifier for $U$ is now asked to send the terms in each pair $\left(t_{i}, s_{i}\right) \in U$ to terms $\sigma\left(t_{i}\right)$ and $\sigma\left(s_{i}\right)$ that are equivalent for $E$ (in symbols, $\sigma\left(t_{i}\right) \approx_{E} \sigma\left(s_{i}\right)$ ).

Once a particular unification problem is known to admit $E$-unifiers, the next task is to find a complete description of its unifiers. For that we first observe that if $\sigma$ is an $E$-unifier for $U$, then $\gamma \circ \sigma$ is also an $E$-unifier for $U$, whenever $\gamma$ is a substitution such that $\gamma \circ \sigma$ is well defined. In this case, we say that $\sigma$ is more general than $\gamma \circ \sigma$. Therefore, a useful way to determine all the unifiers of a particular problem is to calculate a family of unifiers such that any other unifier of the problem is less general

Received June 11, 2012; accepted February 10, 2014

First published online September 12, 2016

2010 Mathematics Subject Classification: Primary 06D15; Secondary 08B30, 06D50, 03G10

Keywords: unification type, equational unification, projective algebra, pseudocomplemented lattice, topological duality

C 2016 by University of Notre Dame $\quad 10.1215 / 00294527-3659307$ 